\title{
Virtual Fitting of a Skirt on a Parametric and a Scanned Body Model
}

\author{
Evrim BUYUKASLAN ${ }^{1}$, Simona JEV NIK ${ }^{2}$, Fatma KALAOGLU ${ }^{2}$ \\ ${ }^{1}$ Istanbul Bilgi Universitesi, Fashion Design, Haciahmet Mahallesi, Pir Hüsamettin Sokak \\ No: 2034440 Beyoğlu, Istanbul, Turkey \\ ${ }^{2}$ Istanbul Technical University, Textile Technology and Design Faculty, İnönü Caddesi, \\ No: 65, Gümü̈ssuyu 34437 Beyoğlu, İstanbul, Turkey
}

\begin{abstract}
Virtual prototyping is often used for animations and graphics; however it also has great advantages for garment industry such as minimizing the time and customization of the garment for individual customers. $3 \mathrm{D}$ body scanners offer better simulation results than a parametric body model. The aim of this study is to see how a garment is simulated on a scanned body model and a parametric body model. In addition we wanted to see the real fit of a skirt on a wearer and compare it with its virtual fitting. Thus we generated two virtual body models: a scanned and a parametric model. Meanwhile we determined a skirt design and created 2D patterns according to the design. Finally the skirt is tailored and generated 2D patterns are used to develop 3D virtual garments. It is well-known that mechanical properties of a fabric have influence on the simulation results. Thus mechanical properties of the fabric are measured by using Fabric Analysis by Simple Testing (FAST) and these data are utilized to generate a simulated skirt. At the end live model is dressed with produced skirt and photographed. Real garment fit and their virtual fits are compared to see how satisfactory the garment simulations are. Currently there are many studies to improve the virtual garment images. This study helps to understand the fit differences between garment simulations on a scanned body model and a parametric body model as well as a chance to compare them with the real fit on a live model.
\end{abstract}

Keywords: Virtual garment simulation, 3D body scanner, virtual model, fabric mechanical properties.

\section{INTRODUCTION}

Computer technology has been so much into our lives since past decades. Fashion industry also benefits from computer technology as it saves time and eases the production processes. To produce a prototype of the designed garment is an ordinary step for traditional apparel production process. However to produce a prototype prior to the real garment takes time and it is costly. The product development stage is almost three times the consumption stage [5]. In addition mostly garment manufacturers and costumers have distance, sometimes overseas, which limits the evaluation of prototype by the costumer. $3 \mathrm{D}$ garment simulation is a great chance to overcome the mentioned issues of traditional apparel production process. Companies are now adapting their manufacturing processes according to $3 \mathrm{D}$ garment simulations in order to save time and decrease production costs. 3D garment simulation is not only used by garment manufacturers but it also has a great application in the film and game industry as well as virtual garment shopping malls [1]. Electronic retailing pushes apparel retailers to employee
3D garment simulation technologies. However consumers state their dissatisfaction about their electronic purchase as they cannot really feel the fabric and try on the garment [2]. Eventhough some electronic retailers provide some virtual models generated according to body measurements they are not yet customized enough to satisfy customers. 3D body scanning is the most advanced method to create a 3D model [3]. 3D body scanners are very advantageous as it captures the body measurement accurately without any physical contact.

Despite its advantages there are some limitations of 3D body scan. Scanned 3D body is individual thus when a different size body virtual model is required a new body scan should be performed to according to the new size body. To overcome this limitation parametric body models are being studied by some researchers. Parametric body models are generated by altering measurments on a scanned body so that new sized and shaped models can be created without any additional scans [5].

Corresponding Author: Evrim Büyükaslan, Tel: +90 (212) 28561 83, E-mail: evrim.buyukaslan@bilgi.edu.tr Submitted: 15 October 2014, Revised: 04 March 2015, Accepted: 01 July 2015 
Several CAD systems such as Browzwear, OptiTex, Lectra and others have developed some softwares to enable the visualization of virtual garments on these virtual models. Real fabric mechanical properties are important for an accurate simulation. Fabric Analysis by Simple Testing (FAST) or Kawabata Evaluation System (KES) measure mechanical properties of a fabric which then can be input for garment simulation software [2].

In this study we designed and produced an A-line skirt at ankle length. The skirt is fitted by our live model and photographed. At the same time we used a 3D body scanner in order to create a scanned body model and parametric body model. Finally the designed skirt is simulated on the virtual models. This study helps to understand the fit differences between garment simulations on a scanned body model and a parametric body model as well as a chance to compare them with the real fit on a live model.

\section{METHODS}

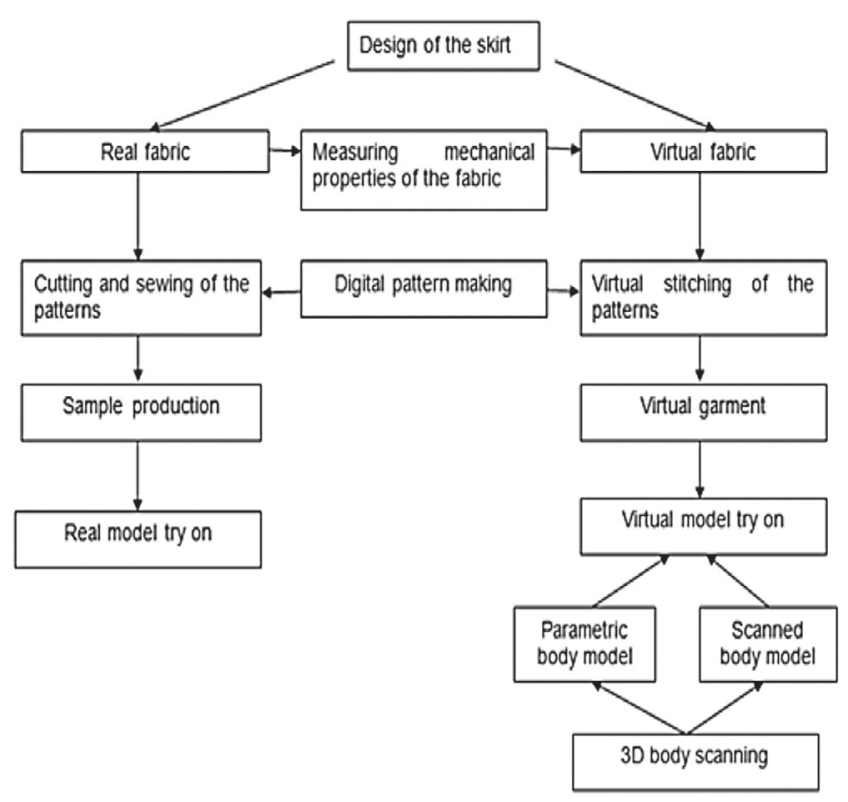

Figure 1. Methodology of the research

We followed several steps during this research. The outline of the research methodology is shown in Figure 1.

Initially we designed the skirt. We preferred a semi fitting skirt in order to see the drapes, wrinkles of the garment and the curves of the body. The skirt is a 6 piece A-line skirt as shown in Figure 2.

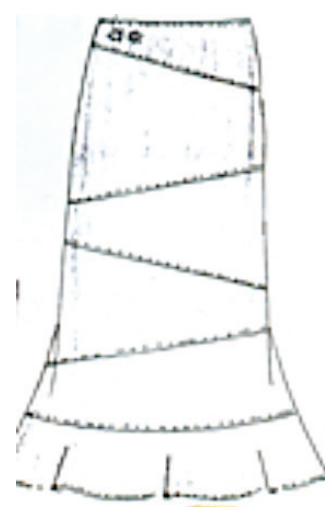

Figure 2. Design of the Skirt

Several pattern pieces enable us to evaluate if the pattern borders are clearly visible in virtual garments. In the next phase of the research, OptiTex CAD system is used to create digital patterns of the skirt.

The produced patterns are used for skirt tailoring and virtual garment generation.

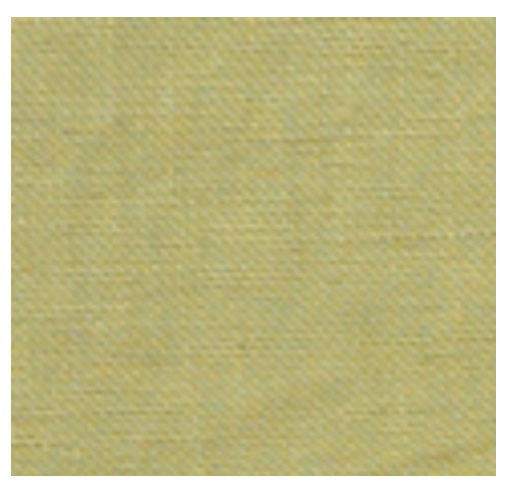

Figure 3. Scanned image of the skirt fabric

Virtual body scan was obtained by a 3D scanner Vitus Smart 3D. The scanned body was reconstructed by MeshLab, Blender and Atos. Our live model was a 55 years old, 42 size women. The virtual body scan was imported into OptiTex PDS program for simulation of virtual garment. In this way, a scanned body model is created and according to scanned body measurements a parametric model is determined in OptiTex program. For creating the parametric model, the measurements are adjusted according to our live model's body measurements.

As a final step, 2D patterns are virtually sewn on virtual models in OptiTex PDS program. 


\section{RESULTS AND DISCUSSION}

The 3D scanned body model and parametric model images are given in Figure 4 and Figure 5 respectively.
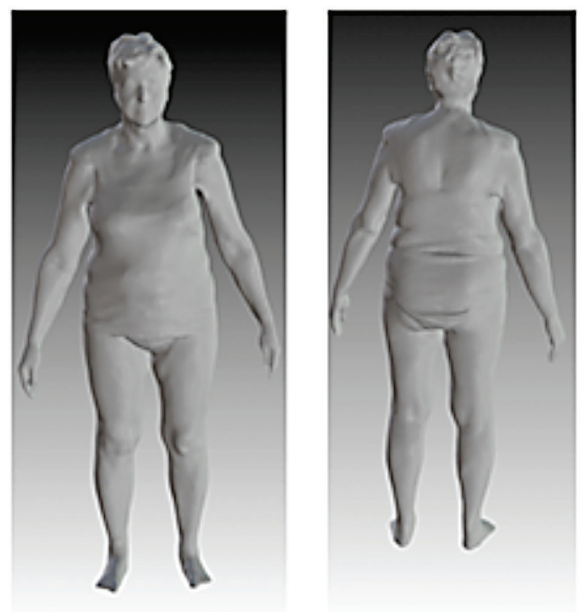

OptiTex

OptiTex

Figure 4. 3D scanned body model

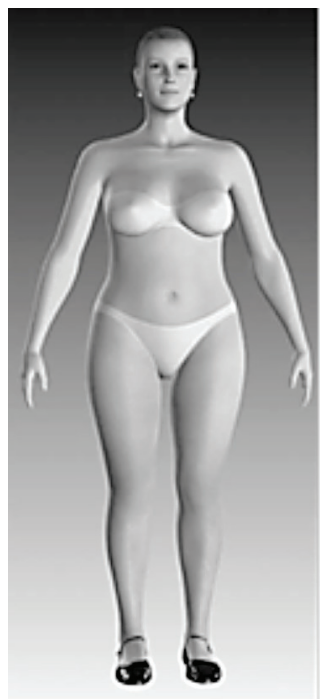

Figure 5. Parametric body model

Parametric body model, scanned body model and the live body represent our tools for observing the skirt fit.

In line with the purpose of this study we compared the fabric simulations on scanned body model parametric body model and live body. Virtual garment simulation on a 3D scanned body model, parametric body model and live body are given in Figure 6, Figure 7 and Figure 8 respectively.

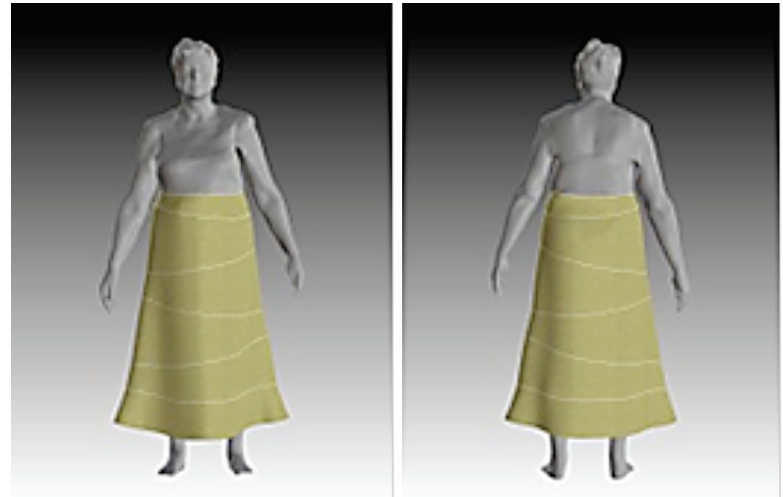

Figure 6. Garment simulations on scanned body model
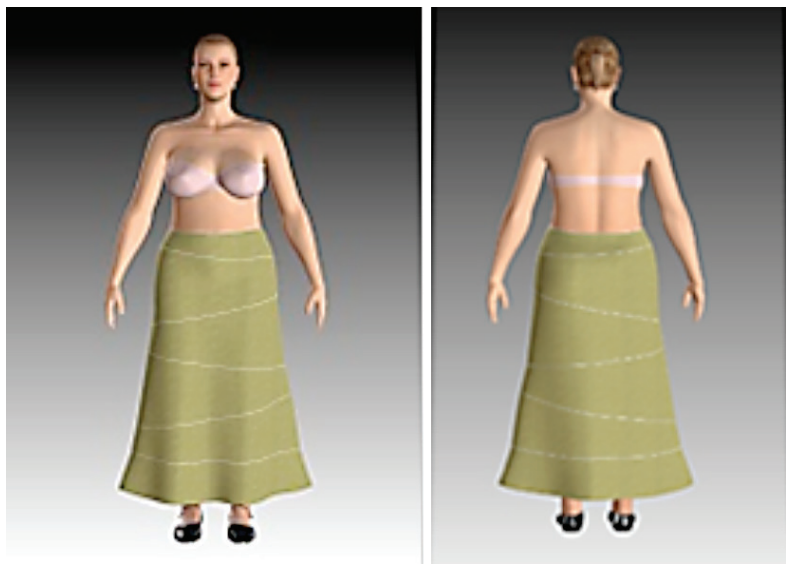

Figure 7. Garment simulations on parametric body model
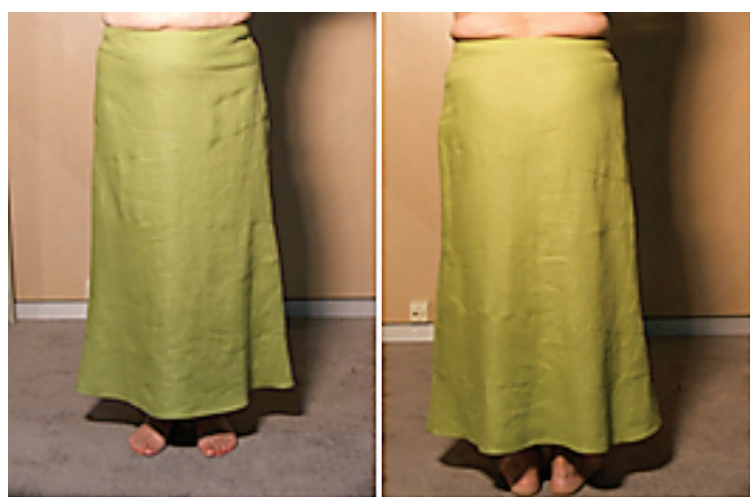

Figure 8. Garment on live body

When we compare the garment simulations on scanned body model, parametric body model and the live body the most significant difference is around the waist area. The skirt waist line is higher for scanned body model. It is almost at breast level. However parametric body model has a more visible waist point as body is idealized and the defects are concealed, so the skirt sits on waist line better than it does on 
scanned body model. However real representation of skirt on live body is considerably different than other two virtual skirts. The deformation of the skin is concealed for virtual models. Real fabric actually deforms according to body shape, both the human skin and fabric reshapes according to the force applied on them. But his property is not well reflected on virtual skirts. Fabric mechanical properties constitute an important role to understand fabric and skin interaction, more investigation on fabric mechanical properties enhances the development of fabric simulations on virtual models. Another difference of real and virtual skirts is hem line. Hem line is actually more draped in real skirt, but especially for back views of the virtual skirts the drapes are not that visible.

A reason for this visual difference might be that the fabric softness is not very well reflected in computer program. Moreover visual skirt on parametric model seems more fitting on hips area. This might be due to the more curvy representation of body on parametric model.

\section{CONCLUSION}

This study helps us to understand the virtual model and virtual garment generating steps. Even if the 3D scanned body models are an identical representation of the corresponding body, it is not very practical to work with scanned bodies. Thus parametric models offer a more commercial approach even though the representations of bodies are now very ideal. The fit differences of a skirt on a live body, scanned and parametric body models are stated in this research. From the images it is concluded that the parametric model and scanned model especially differs at waist and hips areas. This might be a reason of undefined waist line in scanned model and fabric mechanical properties. The fabric and the body behave stiffer then actually they are. To solve this out a further analysis is necessary for computer presentation of fabric properties and their behaviour on parametric and scanned body models.

\section{REFERENCES}

[1] Wong, S. K. (2011). Modeling and simulation techniques for garments. J. Hu (Dü.) inside, Computer technology for textiles and apparel (s. 173-195). Woodhead Publishing Limited.

[2] Istook, C. L., Newcomb, E. A., \& Lim, H. (2011). Three-dimensional (3D) technologies for apparel and textile design. J. Hu (Dü.) inside Computer technology for textiles and apparel (s. 296-320). Woodhead Publishing Limited.

[3] Paquette, S. (1996). 3D scanning in apparel design and human engineering. IEEE Computer Graphics and Applications (16), 11-15.

[4] Rödel, H., Schenk, A., \& Herzberg, C. (2001). Links between design, pattern development and fabric behaviors for clothes and technical textiles. International Journal of Clothing Science and Technology, 217-227.

[5] Kim, S., \& Kyu Park, C. (2004). Parametric body model generation for garment drape simulation. Fibers and Polymers, 5(1), 12-18. 